\title{
Effectiveness of Mobile Health Interventions Promoting Physical Activity and Lifestyle Interventions to Reduce Cardiovascular Risk Among Individuals With Metabolic Syndrome: Systematic Review and Meta-Analysis
}

Irene Sequi-Dominguez ${ }^{1}$, MSc; Celia Alvarez-Bueno ${ }^{1,2}$, PhD; Vicente Martinez-Vizcaino ${ }^{1,3}$, MD; Rubén Fernandez-Rodriguez ${ }^{1,4}$, MSc; Alicia del Saz Lara ${ }^{1}$, MSc; Iván Cavero-Redondo ${ }^{1,2}, \mathrm{PhD}$

${ }^{1}$ Universidad de Castilla-La Mancha, Health and Social Research Center, Cuenca, Spain

${ }^{2}$ Universidad Politécnica y Artística del Paraguay, Asunción, Paraguay

${ }^{3}$ Universidad Autónoma de Chile, Facultad de Ciencias de la Salud, Talca, Chile

${ }^{4}$ Movi-Fitness SL, Universidad de Castilla-La Mancha, Cuenca, Spain

\section{Corresponding Author:}

Celia Alvarez-Bueno, $\mathrm{PhD}$

Universidad de Castilla-La Mancha

Health and Social Research Center

Edificio Melchor Cano Campus Universitario de Cuenca

Camino de Pozuelo, s/n

Cuenca, 16071

Spain

Phone: 34969179100 ext 4659

Email: celia.alvarezbueno@uclm.es

\begin{abstract}
Background: Physical activity and lifestyle interventions, such as a healthy diet, have been proven to be effective approaches to manage metabolic syndrome. However, these interventions require great commitment from patients and clinicians owing to their economic costs, time consumption, and lack of immediate results.

Objective: The aim of this systematic review and meta-analysis was to analyze the effect of mobile-based health interventions for reducing cardiometabolic risk through the promotion of physical activity and healthy lifestyle behaviors.

Methods: PubMed, Scopus, Web of Science, Cochrane Central Register of Controlled Trials, and SPORTdiscus databases were searched for experimental studies evaluating cardiometabolic risk indicators among individuals with metabolic syndrome who were included in technology-assisted physical activity and lifestyle interventions. Effect sizes, pooled mean changes, and their respective 95\% CIs were calculated using the DerSimonian and Laird method. Outcomes included the following clinical and biochemical parameters: body composition (waist circumference [WC] and BMI), blood pressure (systolic blood pressure [SBP] and diastolic blood pressure [DBP]), glucose tolerance (fasting plasma glucose $[\mathrm{FPG}]$ and glycated hemoglobin $\mathrm{A} 1 \mathrm{c}\left[\mathrm{Hb} \mathrm{A}_{1 \mathrm{c}}\right]$ ), and lipid profile (total cholesterol, low-density lipoprotein cholesterol, high-density lipoprotein cholesterol [HDL-C], and triglycerides).
\end{abstract}

Results: A total of nine studies were included in the meta-analysis. Owing to the scarcity of studies, only pooled mean pre-post changes in the intervention groups were estimated. Significant mean changes were observed for BMI $(-1.70 \mathrm{~kg} / \mathrm{m} 2,95 \% \mathrm{CI}$ -3.20 to -0.20 ; effect size: $-0.46 ; P=.03)$, WC $(-5.77 \mathrm{~cm}, 95 \%$ CI -9.76 to -1.77 ; effect size: $-0.54 ; P=.005)$, SBP $(-7.33$ $\mathrm{mmHg}, 95 \% \mathrm{CI}-13.25$ to -1.42 ; effect size: $-0.43 ; P=.02)$, DBP $(-3.90 \mathrm{mmHg}, 95 \% \mathrm{CI}-7.70$ to -0.11 ; effect size: -0.44 ; $P=.04)$, FPG $(-3.65 \mathrm{mg} / \mathrm{dL}, 95 \% \mathrm{CI}-4.79$ to -2.51 ; effect size: $-0.39 ; P<.001)$, and HDL-C (4.19 mg/dL, 95\% CI 2.43-5.95; effect size: $0.23 ; P<.001)$.

Conclusions: Overall, mobile-based health interventions aimed at promoting physical activity and healthy lifestyle changes had a strong positive effect on cardiometabolic risk indicators among individuals with metabolic syndrome. Nevertheless, further research is required to compare this approach with usual care in order to support the incorporation of these technologies in health systems. 
Trial Registration: PROSPERO CRD42019125461; https://tinyurl.com/y3t4wog4.

(J Med Internet Res 2020;22(8):e17790) doi: $\underline{10.2196 / 17790}$

\section{KEYWORDS}

mobile health; mobile technology; telemedicine; metabolic syndrome; physical activity; lifestyle intervention; systematic review; meta-analysis

\section{Introduction}

Metabolic syndrome (MetS) is a cluster of cardiometabolic risk factors that include abdominal obesity, dyslipidemia, hypertension, and insulin resistance [1,2]. MetS has become a worldwide epidemic in parallel with the increase in unhealthy behaviors, such as high rates of physical inactivity and energy dense diets, which have led to alarming obesity prevalence rates in wealthy countries, as well as in developing countries, but to a lesser extent [3]. MetS increases the risk of diabetes mellitus and cardiovascular disease (CVD) in patients with or without a history of cardiovascular events [4]; thus, its early detection may be an important strategy to improve patients' future cardiometabolic risk.

Traditionally, MetS has not been clinically addressed as a single entity but has been managed by treating each of its individual components separately by recommending lifestyle changes (healthy diet and exercise) and pharmacological or even surgical approaches (specifically bariatric surgery, when required). Physical activity interventions have been proven to be effective in reducing CVD risk factors by increasing cardiorespiratory fitness, and dietary interventions have been proven to be effective in decreasing adiposity [5]. In addition, physical activity interventions have been shown to be effective at 12 weeks or more for cardiometabolic parameters [6]. So far, randomized controlled trials (RCTs) of these interventions have required intensive one-on-one or group lifestyle recommendations, raising questions about the feasibility and scalability of implementing these interventions outside of research settings [7].

Mobile-based health (mHealth) technologies can be conceptualized as the remote delivery of health care and exchange of health care information [8]. These technologies can be seen as a complement for some traditional health care methods that, by enabling remote health consultations and monitoring, improve accessibility to health services and the efficiency of some health interventions [8]. Since mobile apps play a key role in everyday life, lifestyle interventions based on these technologies may increase the potential for scalability of interventions and improve their long-term effects and sustainability. In fact, it is expected that the prevention and management of the most common health disorders, which traditionally place a large burden on personnel and resources, will gradually shift to a disease management model in the near future, introducing the use of mHealth [9].

Thus, the aim of this systematic review and meta-analysis was to analyze the effect of lifestyle interventions, including physical activity recommendations through mHealth technologies, on CVD risk factors among individuals with MetS.

\section{Methods}

\section{Design}

This systematic review and meta-analysis was registered in PROSPERO (registration number: CRD42019125461) and was reported following the Preferred Reporting Items for Systematic Reviews and Meta-Analyses (PRISMA) guidelines [10]. The recommendations of the Cochrane Handbook for Systematic Reviews of Interventions [11] were followed to conduct this systematic review and meta-analysis.

\section{Search Strategy}

PubMed (via Medline), EMBASE (via Scopus), Web of Science, Cochrane Central Register of Controlled Trials, and SPORTdiscus databases were searched from their inception to August 2019 following the same PICO (population, intervention, comparison, and outcome) strategy (Figure 1) that included the following: (("metabolic syndrome") AND ("physical activity" OR "lifestyle intervention" OR "health coaching" OR "technology assisted" OR "mobile technology" OR "health technology" OR "internet based" OR "mobile health" OR "mobile phone-based") AND (effectiveness OR utility OR effect OR "cardiometabolic risk factors" OR "cardio-metabolic markers" OR weight OR "body mass index" OR "waist circumference" OR "blood pressure" OR "hemoglobin A1c" OR "fasting plasma glucose" OR "total cholesterol" OR HDL-C OR LDL-C OR triglyceride)). 
Figure 1. PICO (population, intervention, comparison, and outcome) search strategy.

\begin{aligned} & \multicolumn{1}{c}{ Keywords } \\ & \hline Population "metabolic syndrome" \\ & Intervention "physical activity", "lifestyle intervention", "health \\ & coaching", "technology assisted", "mobile technology", \\ & "health technology", "internet based", "mobile health", \\ & "mobile phone-based" \end{aligned}

\section{Selection of Studies}

Eligible articles were experimental studies (RCTs or nonrandomized experimental studies and single-arm pre-post studies), which aimed to measure the effectiveness of lifestyle and physical activity recommendations, using mHealth technologies to reduce cardiometabolic risk factors in individuals with MetS. Studies not written in English or Spanish, including patients with diabetes, or not reporting pre- and postcardiometabolic risk factor values were excluded.

Interventions were classified according to their main characteristics as follows: (1) performing data monitoring or not; (2) carrying out lifestyle and/or physical activity recommendations; and (3) including goal setting tools or not. Outcomes were measured as mean changes in the following cardiometabolic risk indicators: body composition (BMI and waist circumference [WC]), blood pressure (systolic blood pressure $[\mathrm{SBP}]$ and diastolic blood pressure $[\mathrm{DBP}])$, glucose tolerance (fasting plasma glucose [FPG] and glycated hemoglobin $\mathrm{A} 1 \mathrm{c}\left[\mathrm{HbA}_{1 \mathrm{c}}\right]$ ), and lipid profile (total cholesterol, high-density lipoprotein cholesterol [HDL-C], low-density lipoprotein cholesterol [LDL-C], and triglycerides).

The literature search was independently conducted by two reviewers (ISD and ICR), and disagreements were solved by consensus or discussion with a third researcher $(\mathrm{CAB})$.

\section{Data Extraction and Quality Assessment}

The following information was extracted from the included studies: (1) year of publication, (2) country, (3) type of study, (4) sample characteristics (sample size and mean age), (5) intervention characteristics (design and length of intervention), and (6) MetS indicators.

The Cochrane Collaborations tool was used for assessing risk of bias in randomized trials [12], which scores six domains as low, high, or unclear risk. The Quality Assessment Tool for Quantitative Studies [13] was used for nonrandomized experimental and single-arm pre-post studies. It consists of seven domains of risk of bias that are rated as strong, moderate, or weak. Both tools assessed the risk of bias of each study as low (with no high/weak ratings), moderate (with one high/weak rating), or high (with two or more high/weak ratings) [14].
Data extraction and quality assessment were independently performed by two reviewers (ISD and ICR), and inconsistencies were solved by consensus or discussion with a third researcher (CAB). The agreement rate between reviewers was calculated using the kappa statistic.

\section{Statistical Analysis}

The DerSimonian and Laird method [15] was used to compute the pooled mean change estimates for BMI, WC, SBP, DBP, FPG, $\mathrm{HbA}_{1 \mathrm{c}}$, total cholesterol, HDL-C, LDL-C, and triglycerides, with their respective $95 \%$ CIs. Because of the scarcity of RCTs, in which the difference in change between intervention and control groups for the outcome variable was calculated, we calculated the pooled mean pre-post change in the outcome variable for all the interventions (not for the control group). In multiarm trials (two or more intervention groups), we calculated separately the pooled mean pre-post change in each arm, and the common control group was not included in the analysis. Additionally, standardized mean difference scores for the pooled mean change estimates were calculated using the effect size of Cohen $d$, in which the effect was considered weak for values around 0.2 , moderate for values around 0.5 , strong for values around 0.8 , and very strong for values greater than 1.0. When studies reported pre- and postmean values, effect size estimates were calculated for each parameter.

The heterogeneity of results across studies was evaluated using the $I^{2}$ statistic [16]. $I^{2}$ values were assessed as follows: $0 \%-30 \%$, might not be important; 30\%-50\%, moderate heterogeneity; $50 \%-75 \%$, substantial heterogeneity; and 75\%-100\%, considerable heterogeneity. The corresponding $P$ values were also taken into account [11].

Sensitivity analyses were conducted to assess the robustness of the summary estimates and to detect if any particular study accounted for a large proportion of heterogeneity. Random-effects meta-regression models were used to evaluate whether pooled estimates were influenced by the mean age of participants and the percentage of women [17]. Finally, publication bias was evaluated through visual inspection of funnel plots, as well as using the method proposed by Egger [18].

The significance value of the pooled mean change was estimated based on the 95\% CI. Statistical analyses were performed using STATA SE software, version 15 (StataCorp). 


\section{Results}

\section{Systematic Review}

After removing duplicate studies, a total of 47 articles were selected for full-text review following title and abstract screening. Finally, nine studies [19-27] were included in this systematic review (Figure 2).

Of the included studies, five were RCTs [19,23-26] and four were single-arm pre-post studies [20-22,27]. Studies were published between 2013 and 2018, and conducted in four different countries (two in Canada [26,27], one in Germany [23], three in the Republic of Korea [21,22,25], and three in the United States [19,20,24]).

The sample size of the included studies ranged from 12 to 421 participants (51.7\% females, although two studies included men only [21,22]), and the mean age varied between 38.4 and 59.7 years. All participants met the diagnostic criteria for MetS (according to the Adult Treatment Panel III guidelines or the International Diabetes Federation) and were able to access and use the technology required for each intervention.

The interventions were mainly based on physical activity and lifestyle recommendations, with personalization in some cases [20-23], and were delivered through a website, videoconferencing, or an app. The effects of the recommendations were assessed using telemonitoring through mobile devices. In three of the included studies, the interventions were strengthened using self-goal setting tools such as a behavioral strategy for patients to help them visualize their accomplishments and objectives [24,26,27]. The duration of interventions ranged from 8 to 48 weeks, with the number of clinical encounters varying between 2 and 24, and most of them were in-person encounters to perform periodic clinical evaluations (Table 1).

Figure 2. Preferred Reporting Items for Systematic Reviews and Meta-Analyses (PRISMA) diagram.
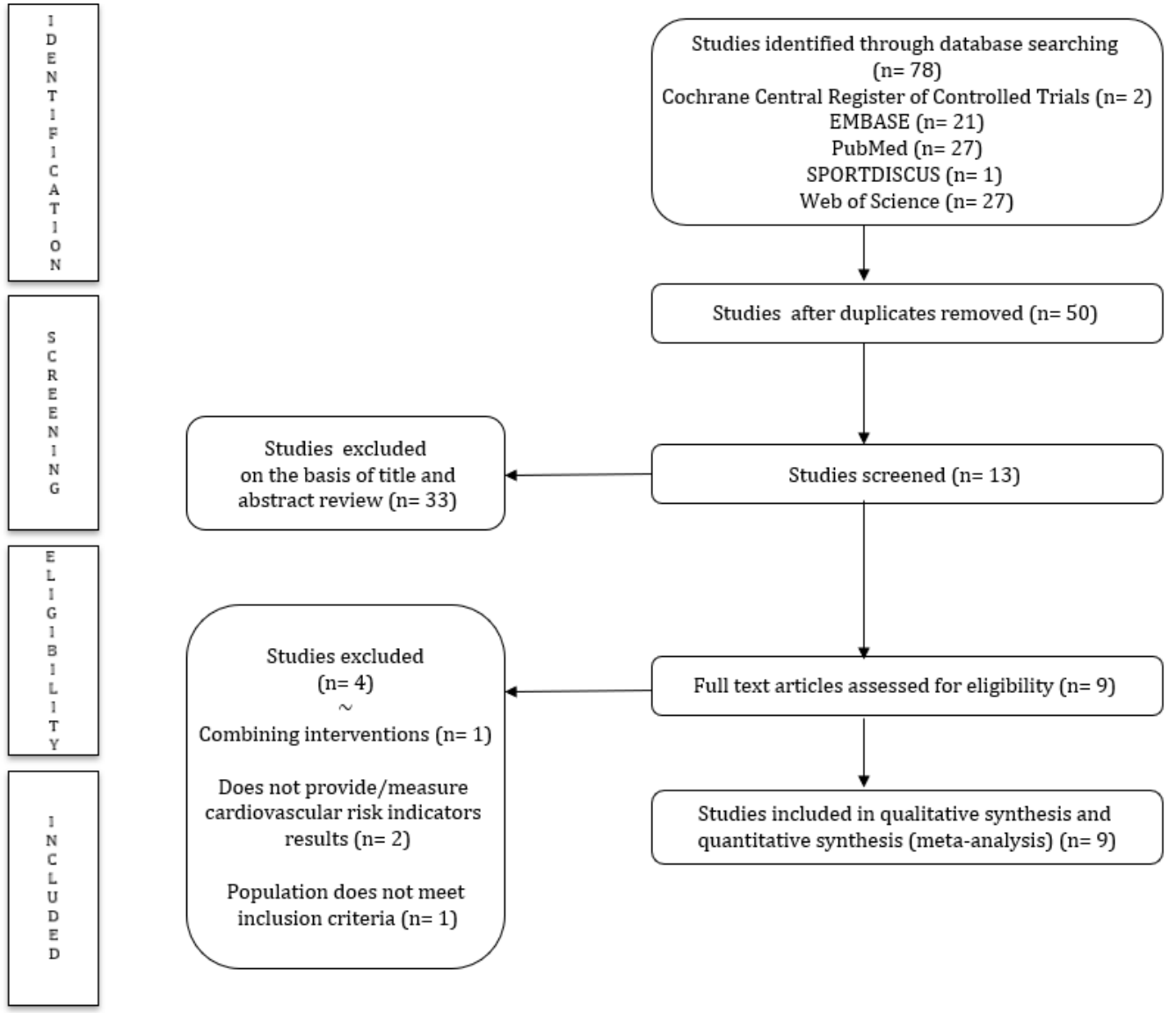
Table 1. Characteristics of the included studies.

\begin{tabular}{|c|c|c|c|c|c|c|c|c|}
\hline \multirow{2}{*}{$\begin{array}{l}\text { First author, } \\
\text { year of publi- } \\
\text { cation }\end{array}$} & \multirow[t]{2}{*}{ Country } & \multirow{2}{*}{$\begin{array}{l}\text { Study } \\
\text { design }\end{array}$} & \multicolumn{6}{|c|}{ Intervention characteristics } \\
\hline & & & $\begin{array}{l}\text { Sample size (n }[\%] \\
\text { female) }\end{array}$ & $\begin{array}{l}\text { Mean age } \\
\text { (years) }\end{array}$ & Intervention design & $\begin{array}{l}\text { Number of } \\
\text { clinical en- } \\
\text { counters }\end{array}$ & Duration & $\begin{array}{l}\text { Cardiometabol- } \\
\text { ic risk outcomes }\end{array}$ \\
\hline $\begin{array}{l}\text { Azar et al, } \\
2016[19]\end{array}$ & USA & $\mathrm{RCT}^{\mathrm{a}}$ & $\begin{array}{l}\mathrm{n}=74(44[59 \%]) \\
\mathrm{IG}^{\mathrm{b}}: \mathrm{n}=37 \\
\mathrm{CG}^{\mathrm{c}}: \mathrm{n}=37\end{array}$ & $\begin{array}{l}59.7(\mathrm{SD} \\
11.2)\end{array}$ & $\begin{array}{l}\text { IG: Data monitoring, } \mathrm{PA}^{\mathrm{d}} \\
\text { and lifestyle web advice, } \\
\text { and weekly videoconfer- } \\
\text { encing } \\
\text { CG: Intervention } 3 \\
\text { months delayed }\end{array}$ & $\begin{array}{l}24 \text { virtual } \\
\text { group sessions } \\
\text { Seven in-per- } \\
\text { son PA ses- } \\
\text { sions }\end{array}$ & 24 weeks & $\begin{array}{l}\text { Weight, weight } \\
\text { change, BMI, } \\
\mathrm{WC}^{\mathrm{e}}, \mathrm{SBP}^{\mathrm{f}}, \\
\mathrm{DBP}^{\mathrm{g}}, \mathrm{TC}^{\mathrm{h}}, \\
\mathrm{HDL}^{\mathrm{i}} \mathrm{C}^{\mathrm{i}}, \mathrm{LDL}- \\
\mathrm{C}^{\mathrm{j}}, \mathrm{TC} / \mathrm{HDL} \text { ra- } \\
\text { tio, and triglyc- } \\
\text { eride }\end{array}$ \\
\hline $\begin{array}{l}\text { Everett et al, } \\
2018 \text { [20] }\end{array}$ & USA & $\begin{array}{l}\text { Pre-post } \\
\text { study }\end{array}$ & $\mathrm{n}=38(24[63 \%])$ & $\begin{array}{l}57.2(\mathrm{SD} \\
9.1)\end{array}$ & $\begin{array}{l}\text { Data monitoring and PA, } \\
\text { weight reduction, and di- } \\
\text { et personalized advice } \\
\text { through a smartphone }\end{array}$ & $\begin{array}{l}\text { Two face-to- } \\
\text { face sessions }\end{array}$ & 12 weeks & $\begin{array}{l}\text { Weight, percent- } \\
\text { age weight } \\
\text { change, BMI, } \\
\text { WC, SBP, } \\
\text { DBP, } \mathrm{HbA}_{1 \mathrm{c}}{ }^{\mathrm{k}}, \\
\text { and } \mathrm{FPG}^{\mathrm{l}}\end{array}$ \\
\hline $\begin{array}{l}\text { Kim and } \\
\text { Kang, } 2013 \\
{[21]}\end{array}$ & $\begin{array}{l}\text { Republic } \\
\text { of Korea }\end{array}$ & $\begin{array}{l}\text { Pre-post } \\
\text { study }\end{array}$ & $\mathrm{n}=18(0[0 \%])$ & $\begin{array}{l}43.1(\mathrm{SD} \\
7.4)\end{array}$ & $\begin{array}{l}\text { PA and weight control } \\
\text { personalized advice } \\
\text { through a website and } \\
\text { SMS text messages }\end{array}$ & $\begin{array}{l}\text { Weekly web } \\
\text { visits }\end{array}$ & 8 weeks & $\begin{array}{l}\text { Weight, visceral } \\
\text { fat mass, WC, } \\
\text { SBP, DBP, } \\
\text { HDL-C, TG, } \\
\text { FPG, and } \\
\text { CVD }^{\mathrm{m}} \text { risk }\end{array}$ \\
\hline $\begin{array}{l}\text { Kim et al, } \\
2014 \text { [22] }\end{array}$ & $\begin{array}{l}\text { Republic } \\
\text { of Korea }\end{array}$ & $\begin{array}{l}\text { Pre-post } \\
\text { study }\end{array}$ & $\begin{array}{l}\mathrm{n}=48(0[0 \%]) \\
\mathrm{IG}: \mathrm{n}=24 \\
\mathrm{CG}: \mathrm{n}=24\end{array}$ & $\begin{array}{l}\text { IG: } 40.88 \\
\text { (SD 7.70) } \\
\text { CG: } 38.38 \\
\text { (SD 6.82) }\end{array}$ & $\begin{array}{l}\text { PA and weight control } \\
\text { personalized advice } \\
\text { through a website and } \\
\text { SMS text messages }\end{array}$ & $\begin{array}{l}\text { Weekly online } \\
\text { sessions }\end{array}$ & 16 weeks & $\begin{array}{l}\text { Weight, body } \\
\text { fat, VFM }{ }^{\mathrm{n}}, \mathrm{WC} \text {, } \\
\text { SBP, DBP, } \\
\text { HDL-C, TG, } \\
\text { FPG, and CVD } \\
\text { risk }\end{array}$ \\
\hline $\begin{array}{l}\text { Luley et al, } \\
2014 \text { [23] }\end{array}$ & Germany & $\mathrm{RCT}$ & $\begin{array}{l}n=178(73[41 \%]) \\
\text { IG1: } n=60(18 \\
[30 \%]) \\
\text { IG2: } n=58(27 \\
[47 \%]) \\
\text { CG: } n=60(28 \\
[47 \%])\end{array}$ & $\begin{array}{l}\text { IG1: } 50.3 \\
(\text { SD } 7.8) \\
\text { IG2: } 50.3 \\
\text { (SD 8.0) } \\
\text { CG: } 50.1 \\
(\text { SD } 8.1)\end{array}$ & $\begin{array}{l}\text { IG1: PA and diet recom- } \\
\text { mendations, data telemon- } \\
\text { itoring, and weekly feed- } \\
\text { back letters } \\
\text { IG2: PA and diet recom- } \\
\text { mendations, data telemon- } \\
\text { itoring, and monthly } \\
\text { feedback calls } \\
\text { CG: PA and diet in-per- } \\
\text { son recommendations }\end{array}$ & $\begin{array}{l}\text { Four in-person } \\
\text { sessions }\end{array}$ & 48 weeks & $\begin{array}{l}\text { Weight loss; } \\
\text { BMI, WC, SBP, } \\
\text { DBP, TC, } \\
\text { HDL-C, LDL- } \\
\text { C, TG, } \\
\text { apolipoprotein } \\
\text { B, uric acid, } \\
\text { alanine amino- } \\
\text { transferase, as- } \\
\text { partate amino- } \\
\text { transferase, } \\
\text { high-sensitivity } \\
\text { CRP }^{\circ}, \text { FPG, } \\
\text { HbA }_{1 \mathrm{c}}, \text { and } \\
\text { HOMA-IR }^{\mathrm{p}}\end{array}$ \\
\hline $\begin{array}{l}\text { Mann et al, } \\
2016 \text { [24] }\end{array}$ & USA & RCT & $\begin{array}{l}n=54(45[83 \%]) \\
\text { IG: } n=27 \\
\text { CG: } n=27\end{array}$ & $\begin{array}{l}\text { IG: } 47.5(\mathrm{SD} \\
11.99) \\
\text { CG: } 43.67 \\
(\mathrm{SD} 9.28)\end{array}$ & $\begin{array}{l}\text { IG: Data monitoring, PA } \\
\text { and diet recommenda- } \\
\text { tions, and goal setting } \\
\text { using electronic medical } \\
\text { records } \\
\text { CG: Traditional recom- } \\
\text { mendations and follow- } \\
\text { up }\end{array}$ & $\begin{array}{l}\text { Two compul- } \\
\text { sory in-person } \\
\text { sessions }\end{array}$ & 24 weeks & $\begin{array}{l}\text { Weight, BMI, } \\
\text { TC, HCL-C, } \\
\text { LDL-C, TG, } \\
\mathrm{HbA}_{1 \mathrm{c}}, \mathrm{REAP-} \\
\mathrm{S}^{\mathrm{q}} \text { score, risk } \\
\text { knowledge, risk } \\
\text { perception, total } \\
\text { step average, } \\
\text { and 7-day step } \\
\text { average }\end{array}$ \\
\hline
\end{tabular}




\begin{tabular}{|c|c|c|c|c|c|c|c|c|}
\hline \multirow{2}{*}{$\begin{array}{l}\text { First author, } \\
\text { year of publi- } \\
\text { cation }\end{array}$} & \multirow[t]{2}{*}{ Country } & \multirow{2}{*}{$\begin{array}{l}\text { Study } \\
\text { design }\end{array}$} & \multicolumn{6}{|c|}{ Intervention characteristics } \\
\hline & & & $\begin{array}{l}\text { Sample size (n [\%] } \\
\text { female) }\end{array}$ & $\begin{array}{l}\text { Mean age } \\
\text { (years) }\end{array}$ & Intervention design & $\begin{array}{l}\text { Number of } \\
\text { clinical en- } \\
\text { counters }\end{array}$ & Duration & $\begin{array}{l}\text { Cardiometabol- } \\
\text { ic risk outcomes }\end{array}$ \\
\hline $\begin{array}{l}\text { Oh et al, } \\
2015[25]\end{array}$ & $\begin{array}{l}\text { Republic } \\
\text { of Korea }\end{array}$ & $\mathrm{RCT}$ & $\begin{array}{l}\text { IG: } n=212(113 \\
[53 \%]) \\
\text { CG: } n=209(99 \\
[47 \%])\end{array}$ & $\begin{array}{l}\text { IG: } 46.78 \\
\text { (SD 13.11) } \\
\text { CG: } 50.35 \\
\text { (SD 14.24) }\end{array}$ & $\begin{array}{l}\text { IG: Body composition } \\
\text { and pedometer data re- } \\
\text { mote monitoring, and } \\
\text { personalized PA and } \\
\text { health online advice } \\
\text { CG: Data records and PA } \\
\text { and diet recommenda- } \\
\text { tions }\end{array}$ & $\begin{array}{l}\text { Four in-person } \\
\text { sessions }\end{array}$ & 24 weeks & $\begin{array}{l}\text { Weight and } \\
\text { BMI }\end{array}$ \\
\hline $\begin{array}{l}\text { Petrella et al, } \\
2014 \text { [26] }\end{array}$ & Canada & RCT & $\begin{array}{l}\text { IG: } n=75(55 \\
[73 \%]) \\
\text { CG: } n=74(56 \\
[76 \%])\end{array}$ & $\begin{array}{l}\text { IG: } 55.7 \text { (SD } \\
10.1) \\
\text { CG: } 57.8 \\
\text { (SD 8.7) }\end{array}$ & $\begin{array}{l}\text { IG: Data telemonitoring, } \\
\text { PA prescription, and goal } \\
\text { setting } \\
\text { CG: PA prescription and } \\
\text { goal setting }\end{array}$ & $\begin{array}{l}\text { Four in-person } \\
\text { sessions }\end{array}$ & 12 weeks & $\begin{array}{l}\text { WC, SBP, } \\
\text { DBP, TC, } \\
\text { HDL-C, LDL- } \\
\text { C, TG, FPG, } \\
\text { HbA }_{1 c}, \\
\text { HOMA-IR, and } \\
\text { high-sensitivity } \\
\text { CRP }\end{array}$ \\
\hline $\begin{array}{l}\text { Stuckey et } \\
\text { al, } 2013 \text { [27] }\end{array}$ & Canada & $\begin{array}{l}\text { Pre-post } \\
\text { study }\end{array}$ & $\mathrm{n}=12(9[75 \%])$ & $\begin{array}{l}56.9(\mathrm{SD} \\
7.0)\end{array}$ & $\begin{array}{l}\text { PA prescription, goal } \\
\text { setting, and data telemon- } \\
\text { itoring }\end{array}$ & $\begin{array}{l}\text { Two in-person } \\
\text { sessions }\end{array}$ & 8 weeks & $\begin{array}{l}\text { WC, SBP, } \\
\text { DBP, TG, } \\
\text { HDL-C, FPG, } \\
\mathrm{VO}_{2} \max ^{\mathrm{r}} \text {, and } \\
\text { steps }\end{array}$ \\
\hline
\end{tabular}

${ }^{\mathrm{a}} \mathrm{RCT}$ : randomized controlled trial.

${ }^{\mathrm{b}} \mathrm{IG}$ : intervention group.

${ }^{\mathrm{c}} \mathrm{CG}$ : control group.

${ }^{\mathrm{d}} \mathrm{PA}$ : physical activity.

${ }^{\mathrm{e}} \mathrm{WC}$ : waist circumference.

${ }^{\mathrm{f}} \mathrm{SBP}$ : systolic blood pressure.

${ }^{\mathrm{g}}$ DBP: diastolic blood pressure.

${ }^{\mathrm{h}}$ TC: total cholesterol.

${ }^{\mathrm{i}} \mathrm{HDL}-\mathrm{C}$ : high-density lipoprotein cholesterol

${ }^{\mathrm{j}}$ LDL-C: low-density lipoprotein cholesterol.

${ }^{\mathrm{k}} \mathrm{HbA}_{1 \mathrm{c}}$ : glycated hemoglobin $\mathrm{A}_{1 \mathrm{c}}$.

${ }^{1}$ FPG: fasting plasma glucose.

${ }^{\mathrm{m}} \mathrm{CVD}$ : cardiovascular disease.

${ }^{\mathrm{n}}$ VFM: visceral fat mass.

${ }^{\mathrm{o}} \mathrm{CRP}$ : C-reactive protein.

PHOMA-IR: homeostatic model assessment of insulin resistance.

${ }^{\mathrm{q}} \mathrm{REAP}-\mathrm{S}$ : rapid eating and activity assessment for patients.

${ }^{\mathrm{r}} \mathrm{VO}_{2}$ max: predicted maximal oxygen capacity.

\section{Risk of Bias}

Seven out of nine studies were assessed as having a high risk of bias (including all single-arm pre-post studies), and the other two were assessed as having a moderate risk of bias. Analyzing

each study individually, all single-arm pre-post studies had the lowest scores in the confounders and blinding domains (Table 2). All RCTs had a high risk of bias in the performance and detection bias domains (Table 3). 
Table 2. Quality assessment of the included pre-post studies.

\begin{tabular}{|c|c|c|c|c|c|c|c|}
\hline $\begin{array}{l}\text { First author, year of } \\
\text { publication }\end{array}$ & Selection bias & Study design & Confounders & Blinding & Data collection & Withdrawals & Risk of bias \\
\hline $\begin{array}{l}\text { Everett et al, } 2018 \\
{[20]}\end{array}$ & Moderate & Moderate & Weak & Weak & Weak & Strong & High \\
\hline $\begin{array}{l}\text { Kim and Kang, } 2013 \\
\text { [21] }\end{array}$ & Moderate & Moderate & Weak & Weak & Strong & Strong & High \\
\hline $\begin{array}{l}\text { Stuckey et al, } 2013 \\
\text { [27] }\end{array}$ & Moderate & Weak & Weak & Weak & Strong & Strong & High \\
\hline
\end{tabular}

Table 3. Quality assessment of the included randomized controlled trials.

\begin{tabular}{|c|c|c|c|c|c|c|c|}
\hline $\begin{array}{l}\text { First author, year of } \\
\text { publication }\end{array}$ & Selection bias & $\begin{array}{l}\text { Performance } \\
\text { bias }\end{array}$ & Detection bias & Attrition bias & Reporting bias & Other bias & Risk of bias \\
\hline Azar et al, 2016 [19] & Low & High & Unclear & Low & Low & Low & Moderate \\
\hline Luley et al, 2014 [23] & Unclear & High & High & Low & Unclear & Low & High \\
\hline Mann et al, 2016 [24] & High & High & High & Low & Low & Unclear & High \\
\hline Oh et al, 2015 [25] & Low & Unclear & Unclear & Low & Low & Low & Low \\
\hline $\begin{array}{l}\text { Petrella et al, } 2014 \\
{[26]}\end{array}$ & High & High & High & Low & Low & Unclear & High \\
\hline
\end{tabular}

\section{Meta-Analysis}

Because of the small number of RCTs, only pooled effect estimates were calculated for mHealth promoting physical activity and lifestyle interventions in pre-post studies. The pre-post pooled mean changes with their heterogeneity statistics for each outcome category are presented below.

\section{Body Composition}

The mean changes were $-1.70 \mathrm{~kg} / \mathrm{m}^{2}(95 \% \mathrm{CI}-3.20$ to -0.20 ; effect size: -0.46$)$ for BMI and $-5.77 \mathrm{~cm}(95 \% \mathrm{CI}-9.76$ to -1.77; effect size: -0.54$)$ for WC. All pooled estimates showed moderate to substantial heterogeneity (BMI: $I^{2}=58.3 \%$; WC: $I^{2}=71.5 \%$ ) (Figure 3). 
Figure 3. Forest plot of meta-analysis of mean changes and effect sizes for body composition parameters. WC: waist circumference.

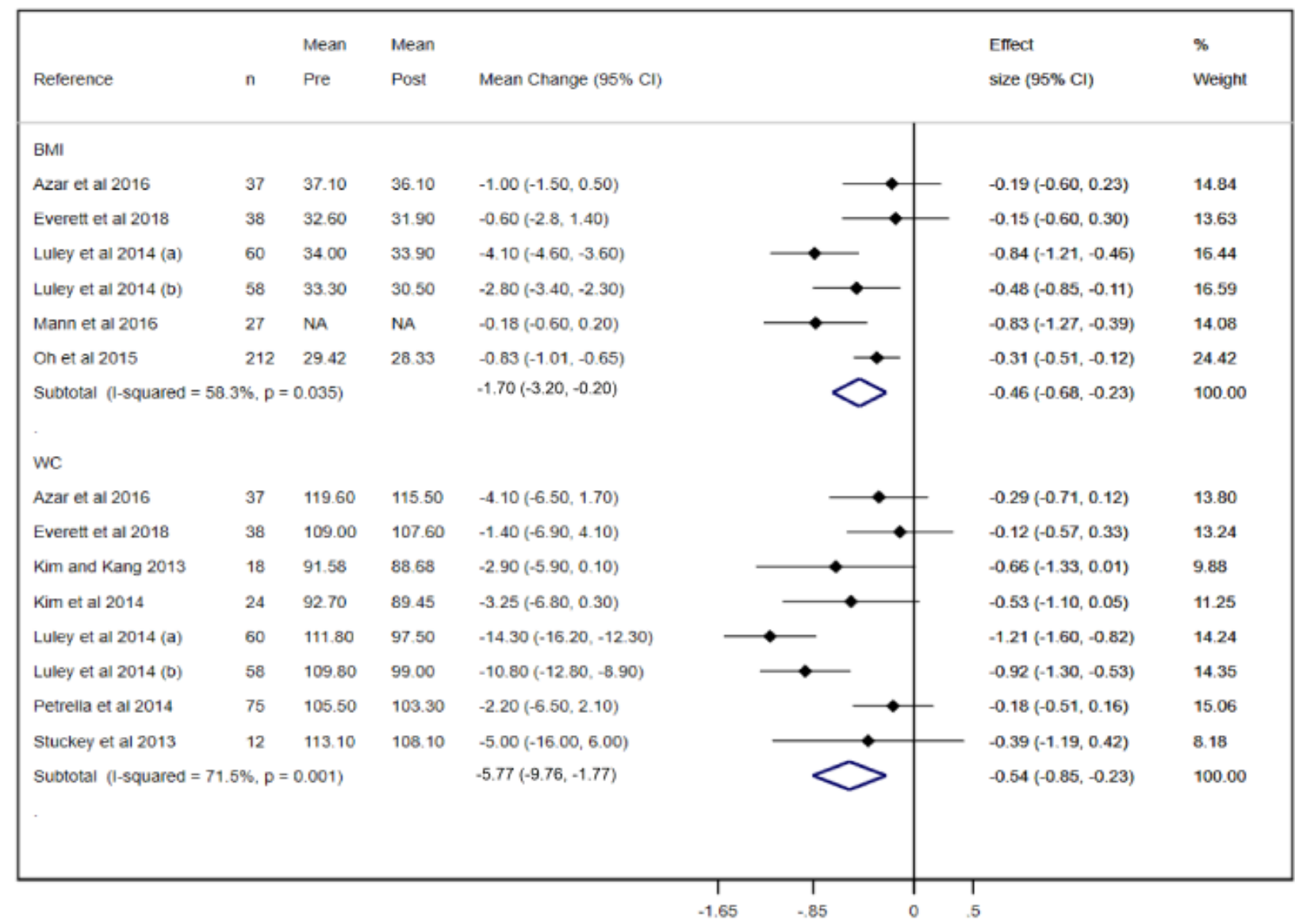

\section{Blood Pressure}

The mean changes were $-7.33 \mathrm{mmHg}(95 \% \mathrm{CI}-13.25$ to -1.42 ; effect size: -0.43$)$ for SBP and $-3.90 \mathrm{mmHg}(95 \% \mathrm{CI}-7.70$ to
-0.11; effect size: -0.44) for DBP, with substantial heterogeneity for $\operatorname{SBP}\left(I^{2}=75 \%\right)$ and DBP $\left(I^{2}=69 \%\right)$ (Figure 4$)$. 
Figure 4. Forest plot of meta-analysis of mean changes and effect sizes for blood pressure parameters. DBP: diastolic blood pressure; SBP: systolic blood pressure.

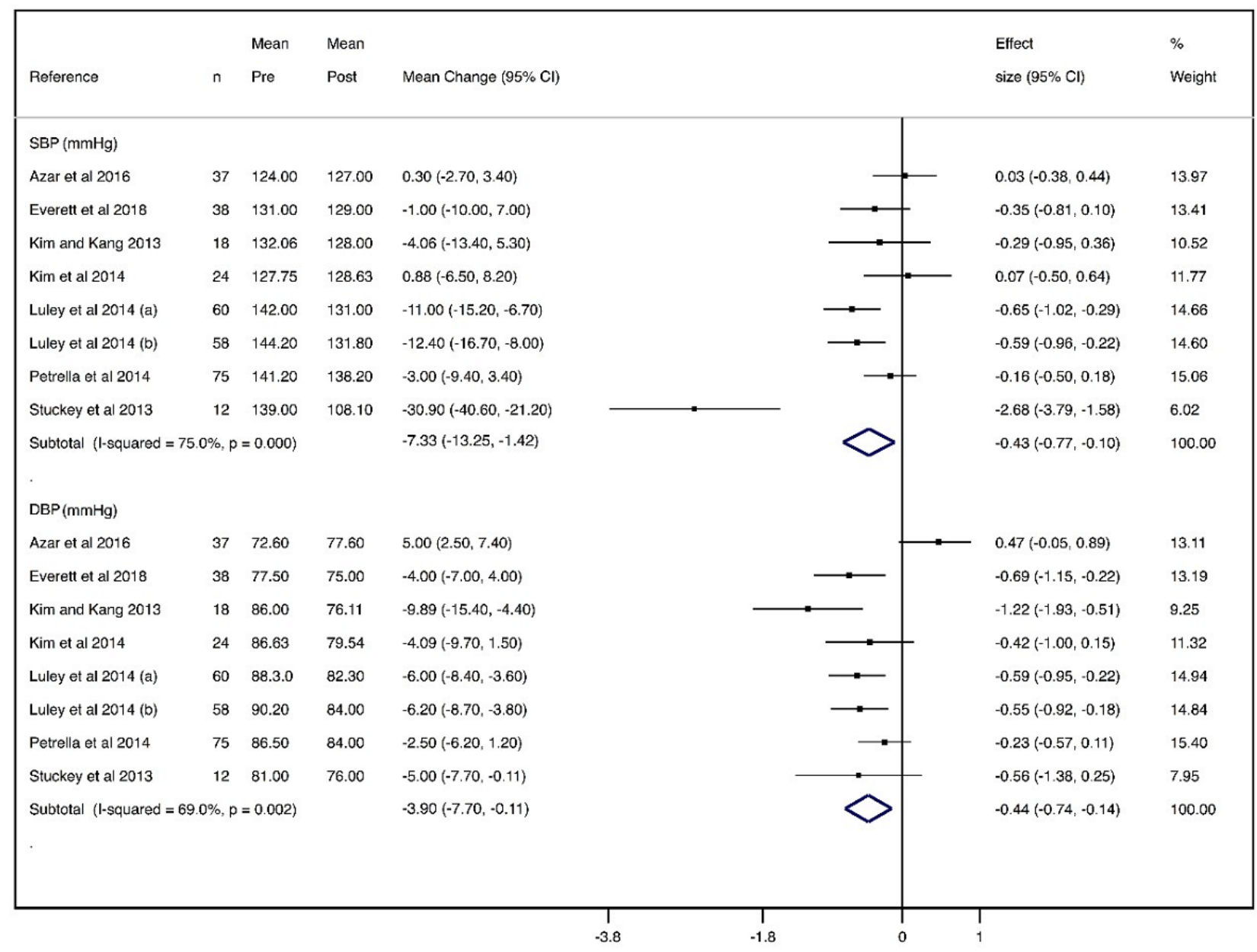

\section{Glucose Tolerance}

The mean changes were $-2.18 \mathrm{mg} / \mathrm{dL}(95 \% \mathrm{CI}-4.41$ to 0.06 ; effect size: -0.35 ) for $\mathrm{HbA}_{1 \mathrm{c}}$, with considerable heterogeneity
$\left(I^{2}=86.7 \%\right)$, and $-3.65 \mathrm{mg} / \mathrm{dL}(95 \% \mathrm{CI}-4.79$ to -2.51 ; effect size: -0.39$)$ for FPG, with substantial heterogeneity $\left(I^{2}=71.5 \%\right)$ (Figure 5). 
Figure 5. Forest plot of meta-analysis of mean changes and effect sizes for glucose tolerance parameters. FPG: fasting plasma glucose; $\mathrm{HbA}_{1 \mathrm{c}}$ : glycated hemoglobin $\mathrm{A}_{1 \mathrm{c}}$.

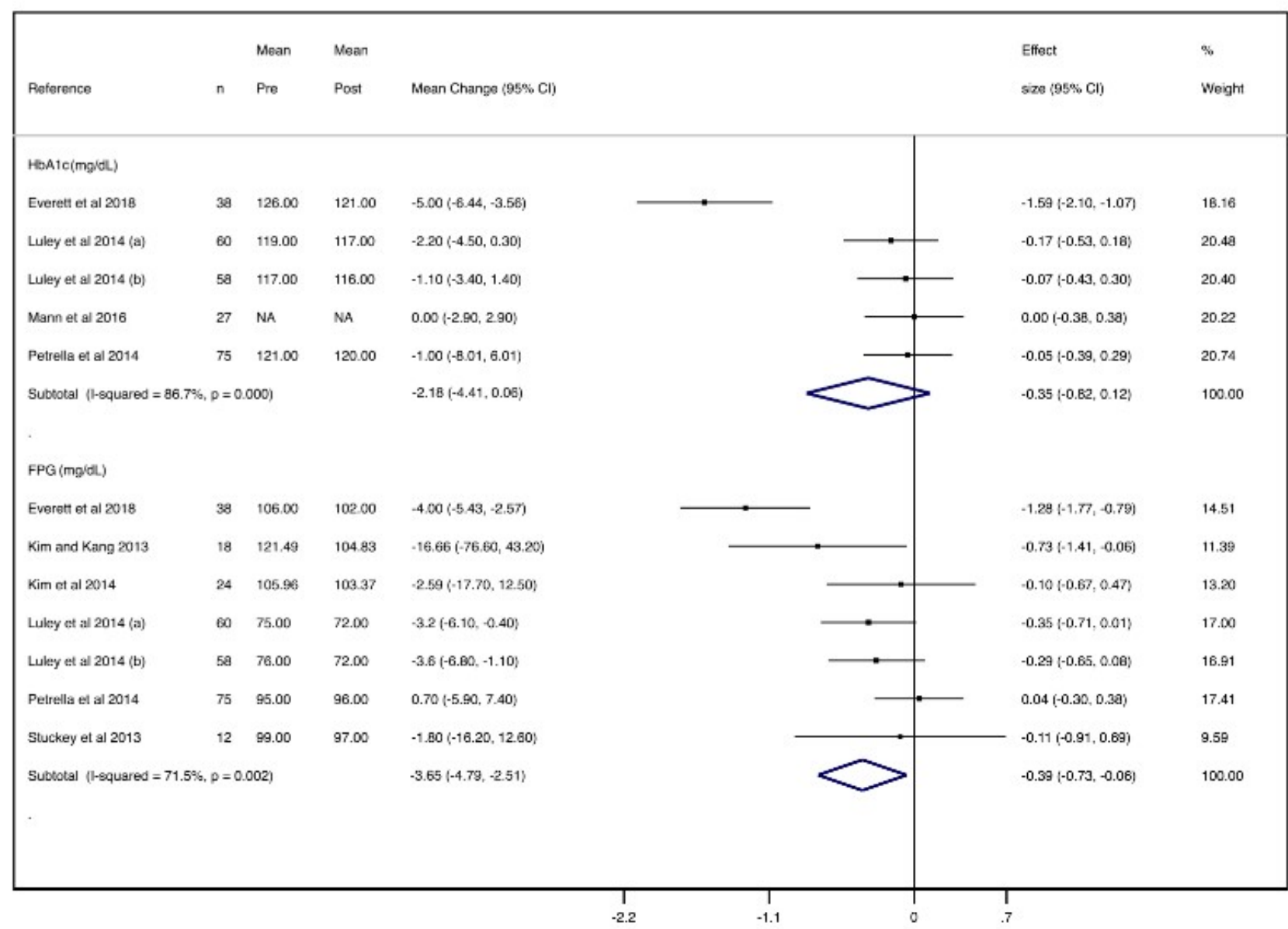

\section{Lipid Profile}

The mean changes were $-3.03 \mathrm{mg} / \mathrm{dL}$ ( $95 \% \mathrm{CI}-10.94$ to 4.89 ; effect size: -0.06$)$ for total cholesterol, with no heterogeneity $\left(I^{2}=0.0 \%\right),-1.85 \mathrm{mg} / \mathrm{dL}$ ( $95 \% \mathrm{CI}-5.93$ to 2.22 ; effect size: $-0.04)$ for LDL-C, with no heterogeneity $\left(I^{2}=0.0 \%\right)$, and -14.03 $\mathrm{mg} / \mathrm{dL}$ (95\% CI -28.20 to 0.13 ; effect size: -0.20 ) for triglycerides, with no heterogeneity $\left(I^{2}=0.0 \%\right)$. Pooled mean changes were not relevant for any of the lipid parameters, except for HDL-C, which increased $4.19 \mathrm{mg} / \mathrm{dL}$ (95\% CI 2.43-5.95; effect size: 0.23$)$, with no heterogeneity $\left(I^{2}=0.0 \%\right)$ (Figure 6). 
Figure 6. Forest plot of meta-analysis of mean changes and effect sizes for lipid profile parameters. HDL-C: high-density lipoprotein cholesterol; LDL-C: low-density lipoprotein cholesterol.

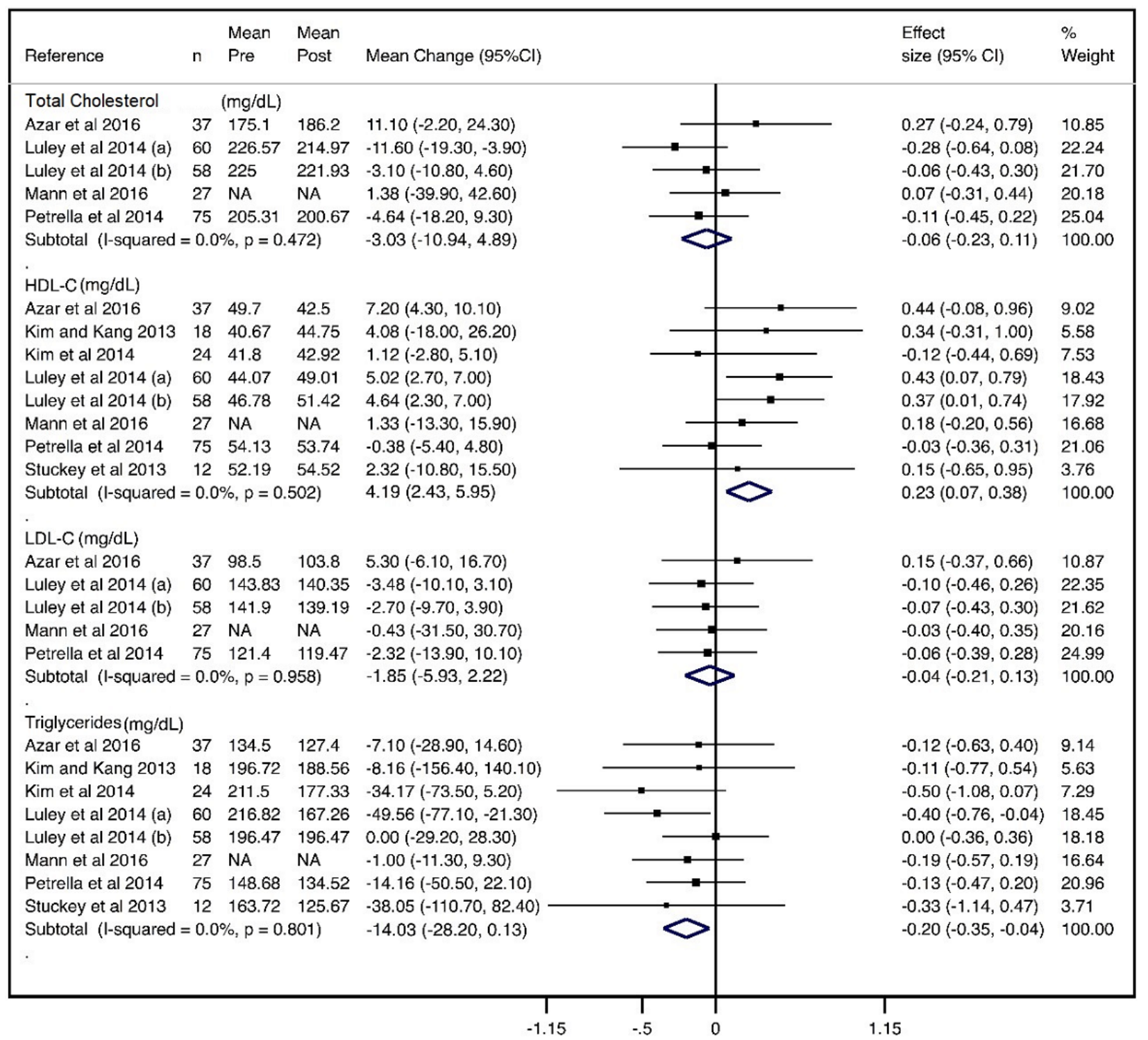

\section{Sensitivity Analysis}

Studies were removed from the analysis one at a time in order to examine their individual impact on the pooled estimates. The pooled mean change of FPG was only significantly modified after removing the study by Everett et al [20] $(-3.04 \mathrm{mg} / \mathrm{dL}$; $95 \% \mathrm{CI}-4.94$ to $-1.15 ; P=.002)$. None of the remaining studies potentially modified the pooled mean change estimate in magnitude or direction.

\section{Meta-Regression}

The random-effects meta-regression models showed that the percentage of females included in the study could influence the pooled estimates of the effect on BMI $(P=.01)$ and triglycerides $(P=.03)$, and the follow-up period could influence the pooled estimates of the effect on WC $(P=.005)$ (Table 4$)$. 
Table 4. Meta-regression findings.

\begin{tabular}{|c|c|c|c|c|c|c|c|c|c|}
\hline \multirow[t]{2}{*}{ Variable } & \multicolumn{3}{|l|}{ Age } & \multicolumn{3}{|c|}{ Percentage of women } & \multicolumn{3}{|c|}{ Follow-up period } \\
\hline & Value, $\mathrm{n}$ & $\beta(95 \% \mathrm{CI})$ & $P$ value & Value, $\mathrm{n}$ & $\beta(95 \% \mathrm{CI})$ & $P$ value & Value, $\mathrm{n}$ & $\beta(95 \% \mathrm{CI})$ & $P$ value \\
\hline \multicolumn{10}{|l|}{ Body composition } \\
\hline BMI $\left(\mathrm{kg} / \mathrm{m}^{2}\right)$ & 6 & $0.04(-0.37$ to 0.45$)$ & .81 & 6 & $\begin{array}{l}0.07(0.03 \text { to } \\
0.12)\end{array}$ & .01 & 6 & $\begin{array}{l}-0.01(-0.03 \text { to } \\
0.01)\end{array}$ & .21 \\
\hline $\begin{array}{l}\text { Waist circumference } \\
(\mathrm{cm})\end{array}$ & 8 & $0.06(-0.68$ to 0.80$)$ & .86 & 8 & $\begin{array}{l}0.01(-0.16 \text { to } \\
0.18)\end{array}$ & .85 & 8 & $\begin{array}{l}-0.02(-0.03 \text { to } \\
0.01)\end{array}$ & .005 \\
\hline \multicolumn{10}{|l|}{ Blood pressure } \\
\hline $\mathrm{SBP}^{\mathrm{a}}(\mathrm{mmHg})$ & 8 & $\begin{array}{l}-0.28(-1.76 \text { to } \\
1.19)\end{array}$ & .65 & 8 & $\begin{array}{l}-0.12(-0.44 \text { to } \\
0.21)\end{array}$ & .41 & 8 & $\begin{array}{l}0.00(-0.04 \text { to } \\
0.04)\end{array}$ & .92 \\
\hline $\mathrm{DBP}^{\mathrm{b}}(\mathrm{mmHg})$ & & $0.49(-0.02$ to 1.01$)$ & .057 & 8 & $\begin{array}{l}0.08(-0.06 \text { to } \\
0.22)\end{array}$ & .23 & 8 & $\begin{array}{l}0.00(-0.02 \text { to } \\
0.02)\end{array}$ & .87 \\
\hline \multicolumn{10}{|l|}{ Glucose tolerance } \\
\hline $\mathrm{HbA}_{1 \mathrm{c}}{ }^{\mathrm{c}}(\mathrm{mg} / \mathrm{dL})$ & 5 & $\begin{array}{l}-0.29(-1.10 \text { to } \\
0.52)\end{array}$ & .34 & 5 & $\begin{array}{l}0.04(-0.08 \text { to } \\
0.16)\end{array}$ & .37 & 5 & $\begin{array}{l}0.01(-0.04 \text { to } \\
0.07)\end{array}$ & .449 \\
\hline $\mathrm{FPG}^{\mathrm{d}}(\mathrm{mg} / \mathrm{dL})$ & 7 & $0.33(-0.12$ to 0.78$)$ & .12 & 7 & $\begin{array}{l}0.08(-0.04 \text { to } \\
0.19)\end{array}$ & .42 & 7 & $\begin{array}{l}0.04(-0.02 \text { to } \\
0.03)\end{array}$ & .75 \\
\hline \multicolumn{10}{|l|}{ Lipid profile } \\
\hline $\begin{array}{l}\text { Total cholesterol } \\
(\mathrm{mg} / \mathrm{dL})\end{array}$ & 5 & $1.58(-1.10$ to 4.26$)$ & .16 & 5 & $\begin{array}{l}0.28(-0.44 \text { to } \\
1.00)\end{array}$ & .31 & 5 & $\begin{array}{l}0.00(-0.02 \text { to } \\
0.02)\end{array}$ & .52 \\
\hline $\mathrm{HDL}^{\mathrm{e}} \mathrm{C}^{\mathrm{m}}(\mathrm{mg} / \mathrm{dL})$ & 8 & $0.24(-0.08$ to 0.56$)$ & .12 & 8 & $\begin{array}{l}0.01(-0.11 \text { to } \\
0.13)\end{array}$ & .80 & 8 & $\begin{array}{l}0.00(0.00 \text { to } \\
0.00)\end{array}$ & .11 \\
\hline LDL-C ${ }^{\mathrm{f}}(\mathrm{mg} / \mathrm{dL})$ & 5 & $0.73(-1.21$ to 2.67$)$ & .32 & 5 & $\begin{array}{l}0.10(-0.34 \text { to } \\
0.53)\end{array}$ & .52 & 5 & $\begin{array}{l}0.00(-0.01 \text { to } \\
0.01)\end{array}$ & .75 \\
\hline $\begin{array}{l}\text { Triglyceride } \\
(\mathrm{mg} / \mathrm{dL})\end{array}$ & 8 & $0.74(-2.97$ to 4.45$)$ & .64 & 8 & $\begin{array}{l}0.49(0.05 \text { to } \\
0.94)\end{array}$ & .03 & 8 & $\begin{array}{l}0.00(-0.01 \text { to } \\
0.01)\end{array}$ & .99 \\
\hline
\end{tabular}

${ }^{\mathrm{a}} \mathrm{SBP}$ : systolic blood pressure.

${ }^{\mathrm{b}} \mathrm{DBP}$ : diastolic blood pressure.

${ }^{\mathrm{c}} \mathrm{HbA}_{1 \mathrm{c}}$ : glycated hemoglobin $\mathrm{A}_{1 \mathrm{c}}$.

${ }^{\mathrm{d}}$ FPG: fasting plasma glucose.

${ }^{\mathrm{e}} \mathrm{HDL}-\mathrm{C}$ : high-density lipoprotein cholesterol.

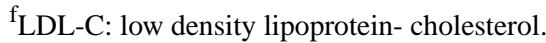

\section{Publication Bias}

After visually examining the funnel plots and performing Egger tests for every parameter (Table 5), publication bias was only significant for WC $(P=.04)$. 
Table 5. Egger test findings.

\begin{tabular}{|c|c|}
\hline Variable & $P$ value \\
\hline \multicolumn{2}{|l|}{ Body composition } \\
\hline BMI $\left(\mathrm{kg} / \mathrm{m}^{2}\right)$ & .98 \\
\hline Waist circumference $(\mathrm{cm})$ & .04 \\
\hline \multicolumn{2}{|l|}{ Blood pressure } \\
\hline $\mathrm{SBP}^{\mathrm{a}}(\mathrm{mmHg})$ & .45 \\
\hline $\mathrm{DBP}^{\mathrm{b}}(\mathrm{mmHg})$ & .58 \\
\hline \multicolumn{2}{|l|}{ Glucose tolerance } \\
\hline $\mathrm{HbA}_{1 \mathrm{c}}^{\mathrm{c}}(\mathrm{mg} / \mathrm{dL})$ & .42 \\
\hline $\mathrm{FPG}^{\mathrm{d}}(\mathrm{mg} / \mathrm{dL})$ & .53 \\
\hline \multicolumn{2}{|l|}{ Lipid profile } \\
\hline Total cholesterol (mg/dL) & .47 \\
\hline 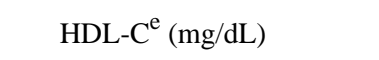 & .31 \\
\hline LDL-C $^{\mathrm{f}}(\mathrm{mg} / \mathrm{dL})$ & .42 \\
\hline Triglyceride (mg/dL) & .24 \\
\hline
\end{tabular}

${ }^{\mathrm{a}} \mathrm{SBP}$ : systolic blood pressure.

${ }^{b}$ DBP: diastolic blood pressure.

${ }^{\mathrm{c}} \mathrm{HbA}_{1 \mathrm{c}}$ : hemoglobin $\mathrm{A}_{1 \mathrm{c}}$.

${ }^{\mathrm{d}}$ FPG: fasting plasma glucose.

${ }^{\mathrm{e}} \mathrm{HDL}-\mathrm{C}$ : high-density lipoprotein cholesterol.

${ }^{f}$ LDL-C: low density lipoprotein- cholesterol.

\section{Discussion}

\section{Principal Findings}

Traditional approaches, such as physical activity programs, brief recommendation interventions, and pharmacological treatments, have been proven to be effective for controlling MetS [28]. However, they are expensive and time-consuming strategies that require a great commitment by both patients and practitioners. Our systematic review and meta-analysis suggested that physical activity and lifestyle interventions based on mHealth technologies are effective for reducing cardiometabolic risk, since they greatly improve body composition, blood pressure, FPG, and HDL-C levels. However, no relevant changes were observed in $\mathrm{HbA}_{1 \mathrm{c}}$, total cholesterol, LDL-C, or triglyceride levels.

Our findings are in line with previous evidence on mHealth interventions in chronic disease patients that reported small to moderate positive effects on primary outcomes, such as cholesterol, weight, and blood pressure [29]. These findings show similar effects both when combining mHealth interventions with usual care (consisting of regular hospital visits, regular visits by primary health care providers at home, or visits to the general practitioner) [30-35] and when mHealth interventions are carried out instead of usual care [36-40]. Such results are consistent with our findings despite the different populations targeted; however, our results show much smaller effect sizes for total cholesterol, LDL-C, and triglycerides, which may be explained by the fewer number of included studies reporting those outcomes.

Among the factors involved in the worldwide increase in sedentary behavior, the use of information and communication technologies and particularly the increase in screen time have been described as the main drivers of low daily energy expenditure [41]. Thus, to involve these technologies as vehicles of preventive interventions could be both a risk and an opportunity. Even though we were unable to demonstrate the superiority of mHealth promoting physical activity and lifestyle interventions over usual care (in-person consultations with clinicians) owing to the scarcity of studies comparing data between control and intervention groups, our results showed that mHealth interventions are effective in improving cardiometabolic risk. Our data regarding the effects of interventions based on mHealth technologies are similar to those involving traditional care [42], suggesting that they could represent an alternative treatment strategy because of their acceptability, scalability, cost-effectiveness, customization, and ability to send time-sensitive messages with an "always on" device [43]. Moreover, mHealth physical activity interventions reduce in-person health provider time and increase self-care by enabling patients to manage their progress [23].

However, our results must be interpreted cautiously, since they are threatened by several limitations that should be acknowledged. First, although a systematic search was carried out through the most well-known databases by two different 
researchers, some scientific contributions reported as grey literature may have been missed in our systematic search. Second, overall, the risk of bias of the included studies was rated as high. Third, there has been some criticism about using single-group studies for evaluating effectiveness [44], and only pre-post estimates for the intervention group could be used because of the scarcity of RCTs reporting the necessary data for control groups. Fourth, there was heterogeneity of interventions owing to differences in components (ie, self-monitoring, type, and persuasiveness of advice), length, and lack of precision in descriptions of the type and intensity of physical activity. Fifth, it was difficult to elucidate whether the outcome changes were due to physical activity or other lifestyle interventions as they were all designed as multicomponent interventions, and hence, it was impossible to isolate each component effect. Sixth, although our results were calculated as pre-post effect sizes, previous literature has recommended avoiding them in meta-analyses [45]. Seventh, the small sample size of some of the included studies diminished their reliability. Eighth, cardiovascular risk parameters were not the main outcomes of most studies. Lastly, none of the included studies used the mHealth evidence reporting and assessment (mERA) checklist, a tool developed by the WHO mHealth Technical Evidence Review Group in order to improve the completeness of reporting mHealth interventions [46]. Despite all of these limitations, our study, as the only updated synthesis evaluating mHealth technologies promoting physical activity and lifestyle interventions to reduce cardiovascular risk in individuals with MetS, establishes a base for future research providing more consistent evidence of their effectiveness.

\section{Conclusion}

Our results show an overall positive effect of physical activity and lifestyle interventions delivered through mobile technologies on MetS indicators, suggesting that they may be effective tools for MetS management. However, further research is needed in order to enable a comparison between the traditional clinical approach and new interventions through mHealth technologies, as these results may be due to the lack of appropriate comparable RCTs because these technologies are novel. Additionally, estimating the independent effect of each component of these interventions would be interesting, and it is important to standardize the implementation of multicomponent interventions in such a way that enough evidence is available for consideration in clinical practice guidelines.

\section{Acknowledgments}

This study was funded by Consejería de Educación, Cultura y Deportes - Junta de Comunidades de Castilla-La Mancha and FEDER funds (SBPLY/17/180501/000533).

\section{Conflicts of Interest}

None declared.

\section{References}

1. Alberti K, Eckel RH, Grundy SM, Zimmet PZ, Cleeman JI, Donato KA, et al. Harmonizing the Metabolic Syndrome. Circulation 2009 Oct 20;120(16):1640-1645. [doi: 10.1161/circulationaha.109.192644]

2. Grundy SM. Metabolic syndrome update. Trends Cardiovasc Med 2016 May;26(4):364-373. [doi: 10.1016/j.tcm.2015.10.004] [Medline: 26654259]

3. Zimmet P, Alberti KG, Shaw J. Global and societal implications of the diabetes epidemic. Nature 2001 Dec 13;414(6865):782-787. [doi: 10.1038/414782a] [Medline: 11742409]

4. Mottillo S, Filion KB, Genest J, Joseph L, Pilote L, Poirier P, et al. The metabolic syndrome and cardiovascular risk a systematic review and meta-analysis. J Am Coll Cardiol 2010 Sep 28;56(14):1113-1132 [FREE Full text] [doi: 10.1016/j.jacc.2010.05.034] [Medline: 20863953]

5. Cornier M, Dabelea D, Hernandez T, Lindstrom R, Steig A, Stob N, et al. The metabolic syndrome. Endocr Rev 2008 Dec;29(7):777-822 [FREE Full text] [doi: 10.1210/er.2008-0024] [Medline: 18971485]

6. Ostman C, Smart NA, Morcos D, Duller A, Ridley W, Jewiss D. The effect of exercise training on clinical outcomes in patients with the metabolic syndrome: a systematic review and meta-analysis. Cardiovasc Diabetol 2017 Aug 30;16(1):110 [FREE Full text] [doi: 10.1186/s12933-017-0590-y] [Medline: 28854979]

7. Reis RS, Salvo D, Ogilvie D, Lambert EV, Goenka S, Brownson RC. Scaling up physical activity interventions worldwide: stepping up to larger and smarter approaches to get people moving. The Lancet 2016 Sep;388(10051):1337-1348. [doi: 10.1016/s0140-6736(16)30728-0]

8. Craig J, Petterson V. Introduction to the Practice of Telemedicine. J Telemed Telecare 2016 Jun 24;11(1):3-9. [doi: $10.1177 / 1357633 \times 0501100102]$

9. Chow CK, Ariyarathna N, Islam SM, Thiagalingam A, Redfern J. mHealth in Cardiovascular Health Care. Heart Lung Circ 2016 Aug;25(8):802-807. [doi: 10.1016/j.hlc.2016.04.009] [Medline: 27262389]

10. Moher D, Liberati A, Tetzlaff J, Altman DG, PRISMA Group. Preferred reporting items for systematic reviews and meta-analyses: the PRISMA statement. Ann Intern Med 2009 Aug 18;151(4):264-9, W64. [doi: 10.7326/0003-4819-151-4-200908180-00135] [Medline: 19622511]

11. Higgins J, Green S. Cochrane Handbook for Systematic Reviews of Interventions Version 5.1.0. Cochrane Collaboration. 2011. URL: https://handbook-5-1.cochrane.org/ [accessed 2019-11-08] 
12. Higgins JP, Altman DG, Gøtzsche PC, Jüni P, Moher D, Oxman AD, Cochrane Bias Methods Group, Cochrane Statistical Methods Group. The Cochrane Collaboration's tool for assessing risk of bias in randomised trials. BMJ 2011 Oct 18;343:d5928 [FREE Full text] [doi: 10.1136/bmj.d5928] [Medline: 22008217]

13. Thomas BH, Ciliska D, Dobbins M, Micucci S. Quality assessment tool for quantitative studies. National Collaborating Centre for Methods and Tools. 2008. URL: https://www.nccmt.ca/knowledge-repositories/search/14 [accessed 2019-11-08]

14. Armijo-Olivo S, Stiles C, Hagen N, Biondo P, Cummings G. Assessment of study quality for systematic reviews: a comparison of the Cochrane Collaboration Risk of Bias Tool and the Effective Public Health Practice Project Quality Assessment Tool: methodological research. J Eval Clin Pract 2012 Feb;18(1):12-18. [doi: 10.1111/j.1365-2753.2010.01516.x] [Medline: 20698919]

15. DerSimonian R, Laird N. Meta-analysis in clinical trials. Controlled Clinical Trials 1986 Sep;7(3):177-188. [doi: 10.1016/0197-2456(86)90046-2]

16. Higgins JP, Thompson SG. Quantifying heterogeneity in a meta-analysis. Stat Med 2002 Jun 15;21(11):1539-1558. [doi: 10.1002/sim.1186] [Medline: 12111919]

17. Thompson SG, Sharp SJ. Explaining heterogeneity in meta-analysis: a comparison of methods. Statist. Med 1999 Oct 30;18(20):2693-2708. [doi: 10.1002/(sici)1097-0258(19991030)18:20<2693::aid-sim235>3.0.co;2-v]

18. Sterne JA, Egger M, Smith GD. Systematic reviews in health care: Investigating and dealing with publication and other biases in meta-analysis. BMJ 2001 Jul 14;323(7304):101-105 [FREE Full text] [doi: 10.1136/bmj.323.7304.101] [Medline: $\underline{11451790]}$

19. Azar KM, Koliwad S, Poon T, Xiao L, Lv N, Griggs R, et al. The Electronic CardioMetabolic Program (eCMP) for Patients With Cardiometabolic Risk: A Randomized Controlled Trial. J Med Internet Res 2016 May 27;18(5):e134 [FREE Full text] [doi: 10.2196/jmir.5143] [Medline: 27234480]

20. Everett E, Kane B, Yoo A, Dobs A, Mathioudakis N. A Novel Approach for Fully Automated, Personalized Health Coaching for Adults with Prediabetes: Pilot Clinical Trial. J Med Internet Res 2018 Feb 27;20(2):e72 [FREE Full text] [doi: 10.2196/jmir.9723] [Medline: 29487046]

21. Kim C, Kang S. Development and a Pilot Test of an Internet-Based Cardiovascular Risk Reduction Program for Korean Male Workers With Metabolic Syndrome. CIN: Computers, Informatics, Nursing 2013;31(4):157-166. [doi: 10.1097/nxn.0b013e3182812829]

22. Kim C, Schlenk EA, Kang S, Park J. Effects of an internet-based lifestyle intervention on cardio-metabolic risks and stress in Korean workers with metabolic syndrome: a controlled trial. Patient Educ Couns 2015 Jan;98(1):111-119. [doi: 10.1016/j.pec.2014.10.013] [Medline: 25468401]

23. Luley C, Blaik A, Götz A, Kicherer F, Kropf S, Isermann B, et al. Weight loss by telemonitoring of nutrition and physical activity in patients with metabolic syndrome for 1 year. J Am Coll Nutr 2014;33(5):363-374. [doi: 10.1080/07315724.2013.875437] [Medline: 25105874]

24. Mann DM, Palmisano J, Lin JJ. A pilot randomized trial of technology-assisted goal setting to improve physical activity among primary care patients with prediabetes. Prev Med Rep 2016 Dec;4:107-112 [FREE Full text] [doi: 10.1016/j.pmedr.2016.05.012] [Medline: 27413670]

25. Oh B, Cho B, Han MK, Choi H, Lee MN, Kang H, et al. The Effectiveness of Mobile Phone-Based Care for Weight Control in Metabolic Syndrome Patients: Randomized Controlled Trial. JMIR Mhealth Uhealth 2015 Aug 20;3(3):e83 [FREE Full text] [doi: 10.2196/mhealth.4222] [Medline: 26293568]

26. Petrella RJ, Stuckey MI, Shapiro S, Gill DP. Mobile health, exercise and metabolic risk: a randomized controlled trial. BMC Public Health 2014 Oct 18;14:1082 [FREE Full text] [doi: 10.1186/1471-2458-14-1082] [Medline: 25326074]

27. Stuckey MI, Kiviniemi AM, Petrella RJ. Diabetes and technology for increased activity study: the effects of exercise and technology on heart rate variability and metabolic syndrome risk factors. Front Endocrinol (Lausanne) 2013;4:121 [FREE Full text] [doi: 10.3389/fendo.2013.00121] [Medline: 24065952]

28. Dunkley A, Charles K, Gray L, Camosso-Stefinovic J, Davies M, Khunti K. Effectiveness of interventions for reducing diabetes and cardiovascular disease risk in people with metabolic syndrome: systematic review and mixed treatment comparison meta-analysis. Diabetes Obes Metab 2012 Jul;14(7):616-625. [doi: 10.1111/j.1463-1326.2012.01571.x] [Medline: 22284386]

29. Eland-de Kok P, van Os-Medendorp H, Vergouwe-Meijer A, Bruijnzeel-Koomen C, Ros W. A systematic review of the effects of e-health on chronically ill patients. J Clin Nurs 2011 Nov;20(21-22):2997-3010. [doi:

10.1111/j.1365-2702.2011.03743.x] [Medline: 21707807]

30. Bergmo T, Wangberg S, Schopf T, Solvoll T. Web-based consultations for parents of children with atopic dermatitis: results of a randomized controlled trial. Acta Paediatr 2009 Feb;98(2):316-320. [doi: 10.1111/j.1651-2227.2008.01033.x] [Medline: $\underline{18795905]}$

31. Bond GE, Burr R, Wolf FM, Price M, McCurry SM, Teri L. The effects of a web-based intervention on the physical outcomes associated with diabetes among adults age 60 and older: a randomized trial. Diabetes Technol Ther 2007 Feb;9(1):52-59 [FREE Full text] [doi: 10.1089/dia.2006.0057] [Medline: 17316098] 
32. Cho J, Chang S, Kwon H, Choi Y, Ko S, Moon S, et al. Long-term effect of the Internet-based glucose monitoring system on HbA1c reduction and glucose stability: a 30-month follow-up study for diabetes management with a ubiquitous medical care system. Diabetes Care 2006 Dec;29(12):2625-2631. [doi: 10.2337/dc05-2371] [Medline: 17130195]

33. Finkelstein SM, Speedie SM, Potthoff S. Home telehealth improves clinical outcomes at lower cost for home healthcare. Telemed J E Health 2006 Apr;12(2):128-136. [doi: 10.1089/tmj.2006.12.128] [Medline: 16620167]

34. Homko CJ, Santamore WP, Whiteman V, Bower M, Berger P, Geifman-Holtzman O, et al. Use of an internet-based telemedicine system to manage underserved women with gestational diabetes mellitus. Diabetes Technol Ther 2007 Jun;9(3):297-306. [doi: 10.1089/dia.2006.0034] [Medline: 17561800]

35. Kim S, Kim H. Effectiveness of mobile and internet intervention in patients with obese type 2 diabetes. Int J Med Inform 2008 Jun;77(6):399-404. [doi: 10.1016/j.ijmedinf.2007.07.006] [Medline: 17881285]

36. Hee-Sung K. Impact of Web-based nurse's education on glycosylated haemoglobin in type 2 diabetic patients. J Clin Nurs 2007 Jul;16(7):1361-1366. [doi: 10.1111/j.1365-2702.2007.01506.x] [Medline: 17584355]

37. Kwon H, Cho J, Kim H, Song B, Ko S, Lee J, et al. Establishment of blood glucose monitoring system using the internet. Diabetes Care 2004 Feb;27(2):478-483. [doi: 10.2337/diacare.27.2.478] [Medline: 14747232]

38. Shea S, Weinstock RS, Starren J, Teresi J, Palmas W, Field L, et al. A Randomized Trial Comparing Telemedicine Case Management with Usual Care in Older, Ethnically Diverse, Medically Underserved Patients with Diabetes Mellitus. Journal of the American Medical Informatics Association 2006 Jan 01;13(1):40-51. [doi: 10.1197/jamia.m1917]

39. Southard BH, Southard DR, Nuckolls J. Clinical trial of an Internet-based case management system for secondary prevention of heart disease. J Cardiopulm Rehabil 2003;23(5):341-348. [doi: 10.1097/00008483-200309000-00003] [Medline: 14512778]

40. Yoon K, Kim H. A short message service by cellular phone in type 2 diabetic patients for 12 months. Diabetes Res Clin Pract 2008 Feb;79(2):256-261. [doi: 10.1016/j.diabres.2007.09.007] [Medline: 17988756]

41. Middelbeek L, Breda J. Obesity and Sedentarism: Reviewing the Current Situation Within the WHO European Region. Curr Obes Rep 2013 Feb 22;2(1):42-49. [doi: 10.1007/s13679-013-0054-y]

42. Maddison R, Rawstorn JC, Shariful Islam SM, Ball K, Tighe S, Gant N, et al. mHealth Interventions for Exercise and Risk Factor Modification in Cardiovascular Disease. Exercise and Sport Sciences Reviews 2019;47(2):86-90. [doi: 10.1249/jes.0000000000000185]

43. Whittaker R, Borland R, Bullen C, Lin RB, McRobbie H, Rodgers A. Mobile phone-based interventions for smoking cessation. Sao Paulo Med. J 2010;128(2):106-107. [doi: 10.1590/s1516-31802010000200014]

44. Ip S, Paulus JK, Balk EM, Dahabreh IJ, Avendano EE, Lau J. Role of Single Group Studies in Agency for Healthcare Research and Quality Comparative Effectiveness Reviews. National Center for Biotechnology Information, U.S. National Library of Medicine. 2013. URL: https://www.ncbi.nlm.nih.gov/books/NBK121314/ [accessed 2019-11-11]

45. Cuijpers P, Weitz E, Cristea IA, Twisk J. Pre-post effect sizes should be avoided in meta-analyses. Epidemiol Psychiatr Sci 2016 Oct 28;26(4):364-368. [doi: 10.1017/s2045796016000809]

46. Agarwal S, LeFevre AE, Lee J, L'Engle K, Mehl G, Sinha C, WHO mHealth Technical Evidence Review Group. Guidelines for reporting of health interventions using mobile phones: mobile health (mHealth) evidence reporting and assessment (mERA) checklist. BMJ 2016 Mar 17;352:i1174. [doi: 10.1136/bmj.i1174] [Medline: 26988021]

\author{
Abbreviations \\ DBP: diastolic blood pressure \\ FPG: fasting plasma glucose \\ $\mathbf{H b A}_{1 \mathbf{c}}$ : glycated hemoglobin $\mathrm{A}_{1 \mathrm{c}}$ \\ HDL-C: high-density lipoprotein cholesterol \\ LDL-C: low-density lipoprotein cholesterol \\ MetS: metabolic syndrome \\ mHealth: mobile-based health \\ RCT: randomized controlled trial \\ SBP: systolic blood pressure \\ WC: waist circumference
}


Edited by G Eysenbach; submitted 13.01.20; peer-reviewed by UB Johansson, J Arsenijevic, L Grepo; comments to author 12.06.20; revised version received 26.06.20; accepted 07.07.20; published 31.08 .20

Please cite as:

Sequi-Dominguez I, Alvarez-Bueno C, Martinez-Vizcaino V, Fernandez-Rodriguez R, del Saz Lara A, Cavero-Redondo I

Effectiveness of Mobile Health Interventions Promoting Physical Activity and Lifestyle Interventions to Reduce Cardiovascular Risk

Among Individuals With Metabolic Syndrome: Systematic Review and Meta-Analysis

J Med Internet Res 2020;22(8):e17790

URL: http://www.jmir.org/2020/8/e17790/

doi: $\underline{10.2196 / 17790}$

PMID: $\underline{32865503}$

(CIrene Sequi-Dominguez, Celia Alvarez-Bueno, Vicente Martinez-Vizcaino, Rubén Fernandez-Rodriguez, Alicia del Saz Lara, Iván Cavero-Redondo. Originally published in the Journal of Medical Internet Research (http://www.jmir.org), 31.08.2020. This is an open-access article distributed under the terms of the Creative Commons Attribution License (https://creativecommons.org/licenses/by/4.0/), which permits unrestricted use, distribution, and reproduction in any medium, provided the original work, first published in the Journal of Medical Internet Research, is properly cited. The complete bibliographic information, a link to the original publication on http://www.jmir.org/, as well as this copyright and license information must be included. 